\title{
La enseñanza del arte en la escena global. El proyecto colaborativo entre el Centro de Investigaciones Artísticas (CIA) y la Maestría en Estéticas Contemporáneas Latinoamericanas (MECL/UNDAV)
}

\author{
Por: Dra. Gabriela A. Piñero', Universidad Nacional del Centro de la \\ Provincia de Buenos Aires, Argentina y Universidad de Buenos Aires, \\ Argentina \\ Recibido: 2 de noviembre, 2017. \\ Aceptado: 15 de marzo, 2018.
}

\section{Resumen}

Este ensayo analiza la emergencia del Centro de Investigaciones Artísticas (CIA) en la ciudad de Buenos Aires en el año 2009, y su posterior colaboración con la Universidad Nacional de Avellaneda (UNDAV) a través de la Maestría en Estéticas Contemporáneas Latinoamericanas (MECL). La fundación de CIA participa de la emergencia en los años 90 y 2000 de una variedad de programas educativos de diverso grado de formalidad que difundieron en Argentina formatos de enseñanza del arte ya legitimados a escala global, pero inéditos a nivel local (las clínicas de arte y las residencias). Estos nuevos modelos educativos desplazaron las modalidades tradicionales de enseñanza del arte y buscaron instalar debates sobre las formas de producción, circulación y profesionalización propias de una escena artística globalizada. Firmado en el año 2016, el proyecto colaborativo CIA/UNDAV puede ser analizado a partir de las exigencias, en cuanto formación y profesionalización, que la escena global del arte impone al artista contemporáneo.

\section{Abstract}

Art Education in the Global Scene. The Collaborative Project between the Center for Artistic Research (CIA) and the Master Program in Contemporary Latin American Aesthetics (MECL/UNDAV)

This article analyzes the emergence of the Centro de Investigaciones Artísticas (CIA) in Buenos Aires (Argentina) in 2009 and its later collaboration with the Universidad Nacional de Avellaneda (UNDAV) through the master's degree in Contemporary Latin American Aesthetics (MECL). The creation of CIA is part of the emerging educational programs with varying degrees of formality that occurred during the period 1999-2000. These programs introduced different educational models of art teaching already globally established, but unknown at the local level (crits and art residences) in Argentina. These new educational models also replaced traditional models of art teaching and challenged contemporary forms of art production, circulation and professionalization. The CIA/UNDAV collaborative project (2016) can be analyzed according to the requirements - in terms of education and professionalization - that the global art scene demands of the contemporary artist.

1 Doctora y Magíster en Historia del Arte, graduada de la Universidad Nacional Autónoma de México. Licenciada en Artes, graduada de la Universidad de Buenos Aires. Actualmente es docente e investigadora de la Universidad Nacional del Centro de la Provincia de Buenos Aires (UNICEN) y profesora de posgrado en la Universidad de Buenos Aires (UBA). Contacto: pinero.gabriela@gmail.com.
Gabriela A. Piñero. La enseñanza del arte en la escena global. El proyecto colaborativo entre el Centro de Investigaciones Artísticas (CIA) y la Maestría en Estéticas Contemporáneas Latinoamericanas (MECL/UNDAV). Revista Comunicación. Año 39, volumen 27, número 1, enero a junio, 2018. Instituto Tecnológico de Costa Rica. ISSN: 0379-3974 / e-ISSN1659-3820.
PALABRAS CLAVE:

Educación artística, enseñanza profesional, artes, investigación, creación artística, innovaciones pedagógicas

KEY WORDS:

Art education, vocational education, arts, research, artistic creation, pedagogic innovation 


\section{El arte es un proceso de investigación (...) es un proceso de evolución de una idea, es prácticamente un trabajo científico}

David Lamelas

\section{INTRODUCCIÓN}

Fundado en el año 2009 por Roberto Jacoby, Graciela Hasper y Judi Werthein, el Centro de Investigaciones Artísticas (CIA) apareció en la escena local como uno de los primeros programas de formación artística que introdujo ciertos desplazamientos en la enseñanza del arte, ya legitimados a nivel global, pero entonces inéditos en Argentina. Los diversos cuestionamientos que los tres artistas dirigieron a las instancias formales de educación artística de comienzos de los años 2000 -las Escuelas de Bellas Artes y el recientemente creado, Instituto Universitario Nacional de las Artes (IUNA), hoy Universidad Nacional de las Artes (UNA)- se dirigían principalmente a tratar el desfase entre las modalidades de enseñanza y producción fomentadas desde estos espacios, y los saberes y experiencias que la escena artística contemporánea y la entonces incipiente industria cultural parecían exigir.

Consolidado desde entonces como uno de los espacios más experimentales de producción/reflexión artística a nivel local, en el año 2016 ClA anunció su colaboración con la Maestría en Estéticas Contemporáneas Latinoamericanas (MECL) de la Universidad Nacional de Avellaneda, Argentina (UNDAV). Dirigida por Adrián Cangi y coordinada por Alejandra González, desde su apertura en el año 2014 la maestría se propuso como un programa destinado a la "gestación de pensamiento crítico, de reelaboración de lenguajes artísticos y de entramado con las fronteras de la ciencia, la técnica y la tecnología" (MECL, 2018). Investigar la articulación de "las prácticas de los lenguajes artísticos con una posibilidad de verbalización o de textualización" se presentó como la posibilidad de aunar producción artística y reflexión teórica, entendiendo el ámbito de la escritura también como un espacio de producción creativa en el campo del concepto (González, comunicación personal realizada en 2017). Inscripta en el número creciente de maestrías y doctorados que desde el año 2006 se abrieron en Argentina en las diversas áreas de lo artístico (historia y teoría del arte, estudios culturales y visuales, curaduría, crítica de arte, producción artística), la MECL buscó distinguirse como el primer programa de posgrado que se replanteó la formación artística, a partir de reconocer la dimensión procesual e investigativa de todo proyecto artístico y la importancia creciente que la teoría adquirió en la producción artística.

Las siguientes páginas analizan la emergencia de CIA a partir del interés expresado por sus fundadores en diseñar y generar nuevos espacios formativos adaptados a las singularidades de la producción contemporánea; inquietud enmarcada en un momento de redefinición de la labor y profesionalización artística a escala internacional y de progresiva inscripción de las producciones locales en la escena global. Abordada por autores como Groys (2014, 2016), la red contemporánea de programas educativos, espacios de exhibición y fuentes de financiación marca un desplazamiento en cuanto a la figura tradicional de artista que actualiza y las formas de trabajo creativo que demanda. En este sentido, la colaboración CIA/UNDAV permite ser analizada como la formalización de algunas de las dinámicas de producción y enseñanza artísticas ensayadas en CIA (vigentes hasta entonces en espacios educativos de menor grado de formalidad) y su pleno reconocimiento como configuradoras de las instancias de formación, producción y legitimización contemporánea.

Este ensayo ofrece los resultados parciales de la investigación posdoctoral de la autora titulada "La reformulación de lo contemporáneo artístico. Crítica, curaduría, historia y nuevas plataformas colaborativas en las artes visuales recientes de Argentina y México", financiada por el Consejo Nacional de Investigaciones Científicas y Técnicas (Conicet, Argentina) entre los años 2014 y 2017. En esta investigación se analizaron los diversos procesos e instancias mediante las cuales a fines de los años 90 e inicios del 2000 se reconfiguró el régimen artístico que dominó la comprensión del arte reciente en Argentina y México. 
La enseñanza del arte en la escena global. El proyecto colaborativo entre el Centro de Investigaciones Artísticas (CIA)

y la Maestría en Estéticas Contemporáneas Latinoamericanas (MECL/UNDAV)

\section{ANTECEDENTES DE CIA: DEBATES SOBRE FORMACIÓN ARTÍSTICA EN ARGENTINA EN TORNO A LOS AÑOS 2000}

Este apartado presenta muy sintéticamente los antecedentes centrales de CIA referidos por sus fundadores. Es necesario realizar una cartografía más exhaustiva de los espacios y programas que en estos años se abocaron a la enseñanza del arte y difusión de nuevos formatos de producción artística que incluya los debates que los orientaban, así como la revisión de las articulaciones y diálogos entre los distintos ámbitos (universitarios, terciarios, programas de menor grado de formalidad, etc.).

En el 2002, Graciela Hasper, Inés Katzenstein y Agustina Cavanagh organizaron una mesa titulada "La formación de los artistas", en la cual se debatió el estado de la formación artística en la Argentina de aquellos años. "La formación de los artistas" fue la segunda mesa realizada en el marco de los Encuentros en el Goethe, gestionados por Inés Katzenstein, Agustina Cavanagh y Graciela Hasper en el año 2002. Las presentaciones y debates generados en estos encuentros fueron posteriormente desgrabados y publicados en la revista ramona (Katzenstein, Hasper y Cavanagh, 2003, pp. 32-59).

Los participantes de la mesa fueron Diana Aisenberg, Guillermo Kuitca, Tulio de Sagastizabal y Luis Fernando Benedit, artistas involucrados en proyectos disímiles que en los años previos al encuentro habían tenido una activa labor en la introducción y legitimación de nuevos formatos en la enseñanza del arte. Iniciada por Guillermo Kuitca en 1991, la Beca Kuitca introdujo el sistema de clínica en Argentina (Pineau, 2012, p. 607). Basado, según Kuitca, en la confianza y diálogo sostenido entre artistas, la clínica de arte planteaba una dinámica pedagógica que -si bien aparecía novedosa en el medio localestaba ya consolidada en programas de formación en el extranjero. Al respecto, Sarah Thornton indaga este formato educativo, conocido como crit, en el Master of Fine Arts del California Institute of the Art (Los Ángeles, Estados Unidos), donde funciona desde los años 1970 (Thornton, 2009, pp. 43-73).

Desde fines de 1980, Diana Aisemberg trabajó con este mismo formato (Katzenstein, Hasper y Cavanagh, 2003, p. 40) y años más tarde coordinó las "Clínicas del Rojas" en el Centro Cultural Rojas de la Universidad de Buenos Aires. Aisemberg fue también quien en gran parte difundió este formato en el interior del país con el apoyo de la Fundación Antorchas. Sobre el formato de la clínica de obra Aisemberg sostiene: "la clínica se basa en un entrenamiento dirigido a presentar la obra y al reconocimiento de 'qué clase de artista soy y quiero ser' y 'de qué se nutre el artista para definir su idea del arte y de su ser artista' a través de preguntas, regalos y trabajos de cooperación" (Katzenstein, Hasper y Cavanagh, 2003, p. 40).

El Taller de Barracas (1994-1995, 1996-1997), del cual Benedit fue tutor junto a Pablo Suárez y Ricardo Longhini, promovió la experimentación con nuevos materiales y formatos de obra. Finalmente, el proyecto Trama -fundado por Leonel Luna, Pablo Zicarello, Marcelo Grossman y Claudia Fontes en 1999- funcionó como un programa internacional de intercambio artístico.

El debate generado en el encuentro, que partía del relato de esas experiencias, reconocía la reconfiguración de los modos de producción, circulación y legitimación en una escena artística que progresivamente se abría a lo global. La distancia con las instancias de educación artística de mayor grado de formalidad, tales como las Escuelas de Bellas Artes, apareció de manera reiterada en la mesa. Hasper señalaba cómo los aportes más relevantes hechos en el campo de la educación en artes visuales habían provenido de espacios no oficiales y lamentaba la ausencia en la mesa de docentes y gestores vinculados a las instancias formativas oficiales, especialmente el IUNA creado a fines de 1996, a la vez que llamaba a una "modernización de los programas".

Por su parte, Kuitca refería la inexistencia de una academia dinámica capaz de participar de manera activa en la formación de artistas, y Ana María Battistozzi denunciaba el sistema decimonónico de talleres que aún regía las Escuelas de Bellas Artes. La verticalidad implícita en la relación maestroalumno, la rígida disciplinariedad de los talleres de grabado, dibujo y pintura, y la falta de articulación entre hacer artístico y pensamiento crítico fueron algunos de los aspectos que -según los participantes- alejaba las instancias de formación artística formales de la producción artística contemporánea (Katzenstein, Hasper y Cavanagh, 2003, p. 45) (se 
analizó algunos de estos desplazamientos en Autor, 2016).

A partir de este diagnóstico sobre el destiempo entre las formas de lo artístico transmitidas en las Escuelas de Bellas Artes y el IUNA -por un ladoy las concepciones que lo artístico adquiría en la escena contemporánea -por otro-, en 2006, Diana Aisenberg, Melina Berkenwald, Marcelo Grossman, Graciela Hasper y Roberto Jacoby gestionaron y coordinaron la primera edición de Residencia Internacional de Artistas en Argentina (RIAA), antecedente directo del $\mathrm{CIA}$.

La presentación del Centro de Investigaciones Artísticas en su sitio web señala las discusiones sobre formación artística, espacios de enseñanza y modelos educativos sostenidas en la primera edición de RIAA, como el germen de un proyecto que demandó tres años en sustancializarse. Según relata Graciela Hasper (2017), la apertura del CIA respondía al deseo de darle permanencia y continuidad a lo que de manera efímera sucedía en las residencias de RIAA. Así, mientras las primeras cuatro ediciones de RIAA (2006-2009) tuvieron lugar en el Viejo Hotel de Ostende, la quinta y última edición (2009) se desarrolló en el recientemente fundado $\mathrm{CIA}$ en la ciudad de Buenos Aires. Cabe destacar que RIAA fue un proyecto autogestionado y financiado con el apoyo de instituciones privadas nacionales e internacionales. Su primera edición (en Ostende, 2006) reunió por 15 días a 24 artistas visuales de entre 25 y 60 años de 12 nacionalidades distintas.

En palabras de Berkenwald (2006), la residencia funcionaba como "un espacio de encuentro entre artistas, y entre uno o varios artistas y un nuevo sitio de trabajo incluyendo a quienes integran dicho medio artístico". Los diálogos e intercambios generados en ese nuevo espacio, a través de la presentación y discusión de proyectos individuales, de actividades conjuntas y la misma experiencia de la convivencia, eran concebidos como elementos que enriquecían la práctica artística. El hecho de desarrollarse durante un período en lugares específicos (y en ocasiones alejados) buscaba sustraer a los participantes de sus obligaciones y distracciones habituales, y fomentar la concentración y continuidad de sus proyectos artísticos. En una suerte de balance sobre el aspecto educativo de esta experiencia, Ber- kenwald remarcó el aprendizaje colectivo surgido de un intercambio entre pares, distinto a la "dinámica alumno-profesor, artista-maestro" (Berkenwald, 2006, p. 39).

La idea de plataforma estuvo presente en cuanto la residencia se reconoció como generadora de proyectos e intercambios que excedían los 15 días de su duración. La inexistencia de talleres desplazaba los espacios habituales de formación y producción artística para privilegiar obras que atravesaban los medios y prácticas tradicionales y se conformaban a la vista de todos. Pinturas, instalaciones, videos, fotografía, intervenciones en el paisaje, performances, textos, muchas bajo la modalidad del site specific (sitio específico), fueron algunas de las piezas exhibidas a la comunidad local al cierre de la residencia.

Si bien experiencias como RIAA se presentaron innovadoras en el medio local de mediados y fines de los años 2000, eran formatos con los cuales los artistas organizadores ya estaban familiarizados. La experiencia de Hasper -la cual refiere su experiencia en la producción de Proyecto Sala 2 en el Centro Cultural Borges (2003) como otro antecedente de RIAA (Hasper, 2017) - y Berkenwald en instituciones y programas artísticos en el exterior, la labor artística y de gestión de Marcelo Grosman a través de Trama, las clínicas coordinadas por Aisemberg, además de los numerosos proyectos desarrollados por Jacoby desde los años 60 en los que la producción, gestión, promoción artística se entrecruzaban y participan en la generación de producciones artísticas híbridas y complejas permitían pensar en una constelación de saberes y experiencias que convergían en la generación de una nueva infraestructura para la formación y producción artística. En un texto que analiza el giro educacional en el arte contemporáneo (educational turn), Lázár refiere aquellos momentos en que ciertas dinámicas de enseñanza experimentales fueron concebidas en términos de práctica artística, desde el trabajo de Joseph Beuys hasta la crítica institucional de los años 80 y 90 (Lázár, 2017). 
La enseñanza del arte en la escena global. El proyecto colaborativo entre el Centro de Investigaciones Artísticas (CIA)

y la Maestría en Estéticas Contemporáneas Latinoamericanas (MECL/UNDAV)

\section{EL CENTRO DE INVESTIGACIONES ARTÍSTICAS (CIA) EN TANTO PLATAFORMA DE PRODUCCIÓN, FORMACIÓN Y PROMOCIÓN ARTÍSTICA}

Ideado como un programa pedagógico gestionado por artistas, CIA empezó sus actividades en agosto de 2009, en un edificio ubicado en la calle Tucumán, 3754/58 de la Ciudad de Buenos Aires, Argentina. Además, tiene base legal en la Fundación Start, responsable de otros proyectos también gestionados por artistas y estrechamente vinculados con el Centro, tales como la revista ramona (2000-2010), la red artística contemporánea Bola de nieve y el proyecto Venus, entre otros (http://www.fundacionstart. org.ar/proyectos).

En su presentación, hecha en la revista ramona, CIA se definió como "un espacio físico y virtual de encuentro y debate entre artistas y pensadores de todo el mundo, en particular de América Latina" (Hasper et al, 2009, p. 9). Con este objetivo y también con el de "proveer instrumentos críticos para la formación y desarrollo de la actividad artística para indagar e intervenir en los cambiantes mapas culturales del momento actual", desde su apertura, CIA convocó un equipo plural de realizadores de cine, teatro, artes visuales y música, filósofos, arquitectos, diseñadores gráficos, escritores y poetas, psicoanalistas, curadores, historiadores y teóricos relevantes de la escena actual. La interdisciplinariedad y la voluntad de hacer del centro un lugar -real y virtual- de debate y difusión de la producción contemporánea signaron en este sentido los inicios de dicho espacio.

Al ser un proyecto autogestionado, la realización de las distintas actividades fue en parte posible gracias a los numerosos apoyos y subsidios que CIA recibió a lo largo de sus años de existencia: Mecenazgo Cultural-Buenos Aires Ciudad, Foundation for Arts Initiatives de Nueva York, laspis International Artists Studio Program en Suecia y programa SIC Subvenciones a la Internacionalización de la Cultura Española (AECID), entre otros. CIA cuenta también con un Club de Amigos y mientras algunas de sus actividades son gratuitas y abiertas al público en general, otras son aranceladas y con cupo limitado.

Junto a la serie de actividades abiertas a la comunidad, ClA diseñó un programa de residencias y un programa de formación de artistas de un año de du- ración -el programa de agentes culturales CIA- que apelaban a convocar e incidir en la comunidad artística local. Si, por un lado, el programa de residencias fomentó el diálogo con realizadores de otros países y buscó dar a conocer las singularidades de la escena local al público internacional, el programa de artistas actualizó las dinámicas y tradiciones que orientaban la formación de los artistas a nivel local. Aunque el número de invitados internacionales se redujo en los últimos años por cuestiones presupuestarias, la heterogeneidad de voces convocadas y su relevancia en los debates artísticos internaciones, manifiesta la voluntad de hacer de CIA un espacio participante de las agendas de producción y debate global. En agosto de 2009 CIA inició sus actividades con una conferencia de Tania Bruguera. Ese mismo año contó con presentaciones de los artistas y curadores Josep-María Martín, Paul Ramírez Jonas, Claire Bishop y Teddy Cruz.

De igual modo, mediante su programa pedagógico, CIA sistematizó algunos de los desplazamientos en cuanto a las formas de producir y enseñar arte ensayadas por las experiencias referidas anteriormente: la importancia creciente que la reflexión teórica adquirió en la formulación de los proyectos artísticos, la interdisciplinariedad, la preeminencia del proceso sobre la obra final, el diálogo horizontal entre pares, formatos de obra tales como la instalación y la performance, que demandan un espectador activo y participativo.

Con un plan de estudios muy flexible y sin objetivos pautados a priori, las diversas actividades realizadas en el marco del programa agentes CIA -del que cada año participan 20 becarios- fomentan una aproximación a lo artístico en tanto práctica de investigación (más que como ámbito productor de objetos mercantiles) abierta a la incertidumbre y experimentación que cruza a través de disciplinas y especialidades (Jacoby, comunicación personal, 2017). Así, las consultorías de proyectos advierten sobre la dimensión procesual e investigativa de las producciones contemporáneas, a la vez que refieren a la concepción ya referida de lo artístico que, según Jacoby (conversación personal, 2017), orienta la labor del Centro. Por su parte, los talleres estimulan la investigación y la experimentación más que la adquisición de destrezas técnicas. Los cursos 
y seminarios profundizan aspectos teóricos e históricos de diversas cuestiones que signaron el arte del pasado y resultan relevantes en la escena actual. Finalmente, las conferencias funcionan como actualizaciones muy concretas sobre debates de la agenda contemporánea.

Si bien las temáticas de talleres y seminarios -así como los docentes- varían año a año, es factible identificar un interés constante en la performance y otras modalidades artísticas vinculadas con la utilización del cuerpo, y en la investigación de los cruces entre arte y política. La denominación de "agentes culturales" a los becarios que se forman en este espacio refiere a la expansión de lo artístico, la disolución de las fronteras entre disciplinas y al vínculo con lo social que CIA promueve con sus diversas intervenciones. En este sentido, lo artístico se actualiza a través del concepto de agencia, como intervención, praxis, acción situada que busca interferir en el vasto campo de lo cultural. Esta concepción de lo artístico arraiga en la propia práctica de Roberto Jacoby, uno de los fundadores de CIA y actual director, reconocido artista de la escena conceptual desde los años 60 (en el año 2011 Hasper renunció a la presidencia de CIA y posteriormente lo hizo también Werthein).

La ruptura disciplinar y la interrelación de medios artísticos, el intercambio y diálogo sostenidos con artistas, teóricos y curadores de diversas latitudes, la investigación y reflexión teórica como parte intrínseca del hacer artístico, pilares de la concepción de práctica artística promovida en CIA, se tradujo en una particular distribución y organización del espacio. Actualmente, CIA funciona en el quinto piso de un edificio ubicado en la calle Bartolomé Mitre 1970 de la Ciudad de Buenos Aires. A través de la recepción se accede a dos grandes salones, también conectados entre sí, con una mesa y sillas. Allí se desarrollan todas las actividades de CIA. Ninguna de las dos locaciones contó con los tradicionales talleres de escultura, grabado y pintura. Salones despojados y pintados de blanco, repletos de sillas constituyeron en ambos edificios los lugares centrales de reunión, convivencia y trabajo.

El edificio de Tucumán contaba también con habitaciones y dependencias donde se alojaban quienes participaban de las residencias artísticas. En el edificio actual, que cuenta con una mediateca abierta al público con más de 1.000 libros (en papel y digitales), los viernes y sábado se desarrollan los seminarios, talleres y laboratorios de la MECL, en los cuales la discusión colectiva sobre los diversos proyectos se constituye en el acto de enseñanza y producción (obra y teoría simultáneamente), desplazando así la enseñanza de la técnica. En estos espacios, se promueve una idea de artista versado en la investigación, el análisis y la reflexión artística, una idea de artista que -si bien reniega de la profesionalización en términos de tecnocratización- está muy al tanto de las discusiones que marcan la agenda global, las instancias legitimadoras de la escena actual y las dinámicas de acceso a financiaciones, becas y subsidios.

\section{FORMALIZACIÓN Y APERTURA: LA COLABORACIÓN CIAVUNDAV}

En abril de 2016, CIA y la UNDAV firmaron un convenio de colaboración por realizarse en el marco de la Maestría en Estéticas Contemporáneas Latinoamericanas. Mientras la maestría se comprometía a gestionar y financiar los seminarios y cursos de posgrado, CIA garantizaba espacio físico para el desarrollo de las diversas actividades, así como su difusión. Durante los años 2014 y 2015 la maestría había desarrollado sus actividades en la Casa del Bicentenario, un espacio con el cual -sin embargo- no mantenía un proyecto académico común (González, comunicación personal, 2017). Iniciados de manera informal con anterioridad, los diálogos e intercambios generados entre ambos espacios ofrecían un beneficio mutuo en cuanto articulaban la estructura, presupuesto y flexibilidad de una universidad joven, con los contactos y legitimidad que en el ámbito del arte actual tenía CIA. En su tesis de maestría sobre la escena artística reciente, Agustina Battezzati analizó la trayectoria de una serie de artistas que exhibieron en el Malba y Mamba entre los años 2010 y 2014, e identificó a CIA como uno de los espacios de formación más elegidos por ellos (Battezzati, 2017).

El reconocimiento que Alejandra González, coordinadora académica de la maestría, hacía de CIA como el lugar actual del arte experimental, junto con el Programa de Artistas de la Universidad Tor- 
La enseñanza del arte en la escena global. El proyecto colaborativo entre el Centro de Investigaciones Artísticas (CIA) y la Maestría en Estéticas Contemporáneas Latinoamericanas (MECL/UNDAV)

cuato Di Tella, dirigido por Katzenstein, partía del diagnóstico (compartido con quienes siete años antes habían fundado CIA) sobre el carácter tradicional (en relación con la enseñanza de los lenguajes artísticos y su funcionamiento) de espacios formales de educación artística, tales como la UNA.

Desmarcándose de la oferta de posgrados en arte existente a nivel nacional, la MECL buscó inaugurar problemáticas nuevas en cuanto a las relaciones arte/política, producción/reflexión y discutir ciertas pautas de configuración de la academia. Desde su propio nombre, la maestría advertía sobre esta nueva delimitación curricular: estética pasaba así a referir al "sistema de evidencias sensibles que revela la existencia de formas de visibilidad en una comunidad en la que se relacionan maneras de hacer con modos de ser".

El estudio del territorio así demarcado ya no correspondía con la indagación (bajo una aproximación histórica) de las disputas y variaciones que definieron diversos abordajes de la estética en la tradición, sino al modo singular que -en el presentecada experiencia artística propone un reparto de lo sensible, una distribución de espacios, tiempos y funciones, y los modos en que los unos y los otros tienen parte en ese reparto. El acontecimiento artístico es reconocido así en su carácter político en cuanto disputa y redistribución de las funciones, espacios y tiempos (Ranciére, 2010). En esta misma dirección, la idea de lo contemporáneo se actualiza como ruptura, acción intempestiva que -justamente por su inadecuación- permite una puesta en crisis, un cuestionamiento del presente (Agamben, 2010). Frente a la imposibilidad de referir, desde el título, configuraciones territoriales como Nuestra América de José Martí, lo latinoamericano recupera el carácter situado de las intervenciones y producciones artísticas. En la línea practicada por $\mathrm{CIA}$, la MECL propone de esta manera una comprensión del pensamiento y la producción estética como acto polítiCO, acción situada.

Concebida como un programa de posgrado dirigido principalmente a artistas, es posible pensar las dinámicas que propone la $\mathrm{MECL}$, dentro de lo que Henk Borgdorff (2006) denomina "investigación en arte". Según el autor, esta perspectiva asume la no "separación de sujeto y objeto, y no contempla ninguna distancia entre el investigador y la práctica artística", y se basa en el presupuesto de que "no existe ninguna separación fundamental entre teoría y práctica en las artes (...) Conceptos y teorías, experiencias y convicciones están entrelazados con las prácticas artísticas y, en parte por esta razón, el arte es siempre reflexivo" (Borgdorff, 2006, p. 10).

Los talleres de CIA funcionan como espacios de experimentación sin derivar, necesariamente, en la producción de obra. En una línea similar, los laboratorios de la MECL se conciben como espacios de investigación y creación, sin una distinción entre teoría y práctica. En la MECL, la tesis final se propone como "un ensayo de interpretación estético-político articulado a una obra en el campo de creación específico" (MECL, 2018).

La idea antes expresada de Bergdorff sobre la no separación entre teoría y práctica en las artes se manifiesta asimismo en el acto de escritura. La crítica dirigida a la escritura académica y el intento de recuperar la tradición ensayística latinoamericana, advierte sobre esta misma voluntad de proponer, desde la interrelación, el ejercicio de escritura y producción de obra como praxis situada. La expresión que Borgdorff recupera de Donald Schön para referir a esta modalidad de práctica artística, "reflexión en la acción", se vincula al modo en que Katzenstein caracteriza la investigación pedagógica conducida en los talleres del programa de Artistas del Di Tella, otro de los programas de formación artística, también fundado en 2009, que González señala como relevantes de la escena actual.

El carácter inaugural en cuanto a las formas de enseñanza y práctica artística actualizadas tanto en CIA como en la MECL se advierte en la desestimación que ambos programas hacen de las instancias y dinámicas de formación artística tradicional. Sobre la selección de los "agentes culturales $\mathrm{CIA}^{\mathrm{A}}$ ", en la que interviene un jurado conformado por artistas, teóricos y curadores nacionales e internacionales, Jacoby sostiene que se priorizan "aquellos participantes con cierta experiencia en sus áreas de trabajo, independientemente de la posesión de títulos formales" (Jacoby, comunicación personal, 2017). Esta desatención reconoce no solo la multiplicidad de formas que asume hoy la producción artística y la pluralidad de trayectorias de sus realizadores, sino 
que también advierte sobre el desfase antes referido entre las concepciones y destrezas fomentadas en las escuelas tradicionales de arte y las modalidades que asume la práctica artística hoy.

El hecho de no requerir títulos oficiales o una formación específica para aplicar al programa de becas hace de sus integrantes un grupo disímil: artistas autodidactas, curadores, actores y directores teatrales, egresados de las carreras de artes y letras, etc. De un modo similar, el ingreso a la MECL no requiere contar necesariamente con títulos oficiales (profesorado o licenciatura), ya que también contempla el ingreso a través de la presentación de portfolio de artista. Esta posibilidad de acceder a un posgrado en artes a través de portfolio que dé cuenta de la producción hasta entonces desarrollada, sin la necesidad de haber transitado los espacios educativos tradicionales, reconoce y a la vez valida la pérdida de prestigio de las instancias formativas oficiales, en cuanto reconoce que el paso por las escuelas de bellas artes ya no es una instancia definitoria para la producción contemporánea.

Como señala Boris Groys (2014), estos desplazamientos dan cuenta de la globalización creciente en las últimas décadas, de "la red de escuelas de arte y de programas educativos" que proponen una "educación estética globalizada y bastante uniforme (...) basada en el mismo canon de vanguardia" (p. 103). La afirmación de Groys (2014, p. 105) de que "la desprofesionalización del arte es en sí una operación altamente profesional" refiere a la transformación en cuanto saberes y habilidades transmitidos en las escuelas de arte. El dominio de la técnica (pintura, escultura, incluso fotografía o video) ya no es definitorio de lo artístico, en un momento en que el arte se comprende también como modo de conocimiento y expansión de los horizontes de vida. El trabajo creativo ya no se concibe, necesariamente, como un trabajo en solitario, sustraído a la mirada e intercambio colectivo o incluso a la conciencia del autor (Groys, 2016, p. 206).

Los procesos de reflexión y producción fomentados a través de los talleres y consultorías de proyectos en CIA y los laboratorios de la MECL hablan en cambio de procesos creativos muy conscientes y sistematizados. La obra resultante ya no será "considerada creativa precisamente porque parece emerger de la nada" (Groys, 2016, p. 206), sino justamente por el trabajo de análisis y reflexión involucrados, por su posibilidad de visibilizar espacios, tradiciones y cuestiones silenciados, y poner en tensión el propio acto de intervención.

\section{CONCLUSIONES}

La escena artística argentina de fines de los años 90 e inicios de los años 2000 estuvo signada por una serie de intervenciones tendientes a reconfigurar el régimen artístico dominante, con el fin de alinear el arte local con los postulados que dominaban la escena global. Junto al despliegue de nuevas estrategias críticas y curatoriales destinadas a romper el mapa artístico dominante desde la segunda posguerra, que jerarquizaba las producciones de diversas partes del globo en centrales y periféricas, originales y derivativas, aparecieron en el medio una variedad de instituciones que funcionaron como agentes de globalización. La aparición de premios, becas y coleccionistas interesados en los nuevos formatos de producción se acompañó de la emergencia de una variedad de programas educativos de diverso grado de formalidad, destinados a difundir nuevas modalidades de enseñanza y producción. Ya legitimadas a escala global, las clínicas de arte, residencias y diversos espacios pedagógicos abocados al arte contemporáneo se fundaban en el diálogo e intercambio colectivo, en la preeminencia del proceso sobre la obra final y la importancia creciente que la reflexión teórica adquirió en la elaboración del proyecto artístico.

Con antecedentes en una serie programas y becas que emergieron en la Argentina en la década de 90, en el año 2009 se fundó el Centro de Investigaciones Artísticas (CIA) en la ciudad de Buenos Aires. Fundado por Graciela Hasper, Judi Werthein y Roberto Jacoby, su apertura respondió a la voluntad de generar un programa educativo capaz de atender las exigencias y singularidades propias de la producción contemporánea, y así propiciar un debate sobre el estado de la educación artística entonces vigente, caracterizada como tradicional y desactualizada por varios artistas y gestores de la época. Con énfasis en la investigación, la instancia procesual y la pesquisa teórica e histórica involucrada en el trabajo artístico, entre sus diversas actividades CIA 
La enseñanza del arte en la escena global. El proyecto colaborativo entre el Centro de Investigaciones Artísticas (CIA)

y la Maestría en Estéticas Contemporáneas Latinoamericanas (MECL/UNDAV)

fundó el Programa de Agentes Culturales CIA, un programa pedagógico de un año de duración que pronto adquirió gran legitimidad y reconocimiento en la escena del arte más reciente a nivel local y regional.

Abierta en el año 2014, la Maestría en Estéticas Contemporáneas Latinoamericanas se fundó en un mismo diagnóstico sobre la inadecuación entre las formas que lo artístico adquiría en la contemporaneidad y las metodologías y enseñanzas practicadas en las instancias más formales de educación artística (las Escuelas de Bellas Artes y la Universidad Nacional de las Artes). El proyecto colaborativo entre la MECL y CIA, firmado en el año 2016, puede -en este sentido- entenderse como una acción destinada a fusionar y potenciar la legitimidad de un programa de posgrado con reconocimiento oficial, con el prestigio de un programa pedagógico experimental gestionado por artistas. La progresiva formalización de algunos de los seminarios y cursos dictados en CIA -en términos de su adecuación a la estructura de seminarios de posgrado- se acompañó de la voluntad -por parte de la MECL- de revisar (abrir y diversificar) ciertos parámetros de definición de la academia. Estas reevaluaciones se apoyaron en una serie de reflexiones teóricas provenientes de la teoría del arte y la educación artística.

El giro político y las tendencias herederas del conceptualismo, dominantes en la escena global del arte desde los años 90 impulsaron estas revisiones en la enseñanza del arte en cuanto fomentaron producciones heterogéneas que -en ocasiones- involucraban importantes y ambiciosas investigaciones teóricas e históricas, y demandaban a los artistas saberes interdisciplinares y la colaboración con profesionales de diversas áreas. La nueva red de premios, becas y subsidios que aparecieron a escala local en esos años exigían, además, formatos de aplicación cercanos a los formatos académicos de presentación de proyectos prevalecientes en las ciencias sociales y humanas. Las reflexiones provenientes del campo de la educación artística y una pedagogía crítica -por otra parte- reconocieron los procesos de investigación y producción de conocimiento involucrados en muchas de estas prácticas y tuvieron que desarrollar formas de evaluación nuevas que desplazaban la noción de "calidad" como criterio de valoración de la producción artística.

Analizar cómo se forman y califican los artistas en la actualidad (García Canclini, 2014, p. 119) se revela así como una instancia necesaria para examinar la reconfiguración del régimen de lo artístico en la contemporaneidad. En este sentido, se abre una serie de preguntas que permitiría avanzar en esta comprensión: ¿cuáles son las diversas trayectorias del artista contemporáneo y cómo estas trayectorias han variado históricamente? ¿Cuáles son las reglas de valoración de las obras en la contemporaneidad y en qué medida las nuevas instancias y espacios de formación y profesionalización se adaptan a ellas y las modelan? ¿Cuáles son las nuevas formas, dinámicas y redes de trabajo artístico? ¿Cómo se conceptualizan los procesos artísticos y cómo se redefinió, en este momento histórico, el concepto de creatividad?

\section{REFERENCIAS BIBLIOGRÁFICAS}

Agamben, G. (2010). ¿Qué es lo contemporáneo? Otra Parte. 20, 77-80.

Autor. (2016). Nuevas instancias de formación y la discusión sobre los modos de hacer arte en la contemporaneidad. Las clínicas de arte en Tandil, 2008-2009. IV Jornadas Internacionales y VII Jornadas Nacionales de Historia, Arte y Política. Facultad de Arte, Universidad Nacional del Centro de la Provincia de Buenos Aires.

Battezatti, A. (2017). La conformación de los valores artísticos contemporáneos. Participación y circulación en el Museo de Arte Moderno de Buenos Aires y en el Museo de Arte Latinoamericano de Buenos Aires. 2010-2014 (Tesis de maestría inédita). IDAES/UNSAM, Argentina.

Berkenwald, M. (2006). Cotidianeidad es en el presente contexto una palabra importante. Revista de artes visuales ramona. 63, 38-45. Disponible en www.ramona.org.ar.

Borgdorff, H. (2006). El debate sobre la investigación en las artes. Recuperado de https://www. gu.se/digitalAssets/1322/1322698_el-debatesobre-la-investigaci--n-en-las-artes.doc. 
Canela, J. (2017). La intensidad turbo. Entrevista a Inés Katzsenstein. Recuperado de http://terremoto.mx/article/la-intensidad-turbo/.

Elkins, J. (2017). Artists with PhDs. Recuperado de http://www.jameselkins.com/yy/.

García Canclini, N. (2014). El mundo entero como lugar extraño. Buenos Aires: Editorial Gedisa.

Groys, B. (2014). Volverse público. La transformación del arte en el ágora contemporánea. Buenos Aires: Caja Negra Editora.

Groys, B. (2016). Arte en flujo. Ensayos sobre la evanescencia del presente. Buenos Aires: Caja Negra Editora.

Hasper, G. (2007). Aires de cambio en Buenos Aires: clínicas, becas y proyectos. Revista de artes visuales ramona, 77, 51.

Hasper, G., Jacoby, R. y Werthein, J. (2009). El Centro de Investigaciones Artísticas. Revista de artes visuales ramona, 93, 9. Disponible en www.ramona.org.ar. Disponible en www.ramona.org. ar.

Katzenstein, I. (2017). ¿Hay arte en la escuela? Recuperado de http://www.coleccioncisneros.org/ es/editorial/debate/contribution/\%C2\%BFhayarte-en-la-escuela.

Katzenstein, I. e Iglesias, C. (Comps). (2017). ¿Es el arte un misterio o un ministerio? El arte contemporáneo frente a los desafíos del profesionalismo. Buenos Aires: Siglo XXI Editores..

Katzenstein, I., Hasper, G. y Cavanagh, A. (2003). La formación de los artistas. Revista de artes visuales ramona, 29-30, 32-58. Disponible en www.ramona.org.ar.

Lázár, E. (2017). Educational turn. Extraído de http:// worker01.e-flux.com/pdf/article_18.pdf.

Pineau, N. (2012). Espacios de exhibición durante los años noventa en Buenos Aires y la formación de una nueva escena artística. En M.I. Baldasarre y S. Dolinko (Eds.). Travesías de la imagen. Historias de las artes visuales en Argentina (607-635). Bs As: UNTREF.

Rancière, J. (2010). El desacuerdo. Estética y política. Bs As: Nueva Visión.
Rogoff, I. (2010). Turning. e-flux \#0. Recuperado de http://worker01.e-flux.com/pdf/article_18.pdf.

Romero, J.C. (2007). Escuelas de arte para la época. Revista de artes visuales ramona, 77, 23-27. Disponible en www.ramona.org.ar.

Sternfeld, N. (2010). Unglamorous Tasks: What Can Education Learn from its Political Traditions? e-flux \#14. Recuperado de http://www.e-flux. com/journal/14/61302/unglamorous-taskswhat-can-education-learn-from-its-politicaltraditions/.

Thornton, S. (2009). Siete días en el mundo del arte. Buenos Aires: Edhasa. 